\title{
Comparison of Analgesic Effects of Epidural Infusion of Fentanyl and Fentanyl-bupivacaine in Spine Surgery: A Randomized Clinical Trial Study
}

\author{
Ali Ashraf ${ }^{1 *}$, Gita Khanjanian ${ }^{1}$, Shahrokh Yousefzadeh-Chabok ${ }^{2}$, Babak Alijani ${ }^{2}$, Neda Ebrahimpour ${ }^{1}$, Tahere \\ Chavoshi ${ }^{1}$ \\ ${ }^{1}$ Poursina Hospital, Guilan University of Medical Sciences, Rasht, Guilan, Iran \\ 2 Guilan Road Trauma Research Center, Poursina Hospital, Guilan University of Medical Sciences, Rasht, Guilan, Iran
}

*Corresponding Author: Poursina Hospital, Guilan University of Medical Sciences, Rasht, Guilan, Iran. Tel: +981333368773, Fax: +981333368773, E-mail: draliashraf@yahoo.fr

\section{Abstract}

Background \& Aim: Although most patients experience severe pain after major spinal fixation surgery, little attention has been paid to control it with a multi-modal approach. The aim of this study is to select appropriate method of pain management after spinal surgery.

Methods \& Materials/Patients: This study was a randomized clinically controlled trial in candidates of spondylolisthesis and spinal fixation surgery under general anesthesia. 90 patients were divided into three groups, as follows: 1) Intervention group of epidural fentanyl (F): patient-controlled analgesia (PCA) pump infused $100 \mu \mathrm{g}$ of fentanyl and $98 \mathrm{cc}$ of normal saline at the rate of $3-5 \mathrm{ml} / \mathrm{h}$ in the epidural space via an epidural catheter placed by surgeon 2) Intervention group of epidural fentanyl-bupivacaine (FB): PCA pump infused $100 \mu \mathrm{g}$ of fentanyl and bupivacaine $0.125 \%$ at the rate of $3-5 \mathrm{ml} / \mathrm{h}$ in the epidural space. 3) IV morphine group (M): No medication was received epidurally. In case of visual analog scale (VAS) score more than four, one mg of IV morphine bolus was administered to patient. The blood pressure, heart rate, respiratory rate and pain severity were recorded at the moment of patient's transfer from recovery room to the ward and compared in three groups every six hours for 24 hours. The results of this study were analyzed by statistical tests including repeated measures ANOVA, Kruskal-Wallis, Fisher's exact test and SPSS software 16. P values less than 0.05 were considered statistically significant.

Results: The results of the study showed that VAS score in fentanyl-bupivacaine group was significantly lower than that in the two other groups during the first 18 hours. However, VAS score in all groups decreased at 24th hour postoperatively (less than three) suggesting that there was no statistically significant difference between the three groups. In this study, no serious and severe complications were observed except for a few cases of nausea in the control group.

Conclusion: Epidural analgesia with fentanyl-bupivacaine is a safe and effective way to postoperative pain management in listhesis and spinal surgeries.

Keywords: Analgesia; Epidural; Listhesis; Fentanyl; Bupivacaine

Please cite this paper as: Ashraf A, Khanjanian G, Yousefzadeh-Chabok Sh, Alijani B, Ebrahimpour N, Chavoshi T. Comparison of Analgesic Effects of Epidural Infusion of Fentanyl and Fentanyl-bupivacaine in Spine Surgery: A Randomized Clinical Trial Study. Iran J Neurosurg. .2015;1(1):28-34

\section{Introduction}

Most patients experience severe pain after major spinal fixation surgery and there is higher intensity of pain in the first 48 to 72 hours postoperatively and then decreases (1-5). The pain severity is often so high that intravenous narcotic analgesics are mostly prescribed for patients during the first two days after surgery. Postoperative severe pain prevents early movement of the patients and increased hospital stay and therefore will result in various complications such as deep vein thrombosis and atelectasis and surgical site infection (1). Evidence suggests that a postoperative pain control improves the outcome of surgical procedures and reduces the morbidity. Several medications are used to manage pain including acetaminophen, non-steroidal 
anti-inflammatory drugs, weak and strong narcotics. These medications are used with a variety of ways such as intravenous, oral, dermal and multi-modal, such as peripheral nerves catheters or neuraxial with or without anesthetics. Benefits of neuraxial methods (such as epidural analgesia method) with local anesthetics include narcotic pain control and reduced systemic narcotics consumption and reduced respiratory depression. Their complications are neurologic abnormalities (6-8). Concomitant use of narcotics in neuraxial method often increases the quality of analgesia, but it can cause pruritus, nausea and urinary retention. Using epidural analgesia in high risk patients undergoing major surgeries leads to better control of pain than intravenous administration (9). PCA pump is used to control pain after spinal surgery (10-12). Little attention has been paid to pain management especially multi-modal approaches in these patients, despite of intense pain after spinal surgery. Continuous epidural infusion method was used in a few studies in which effectiveness of epidural analgesia was demonstrated as to reduce side effects in spinal surgeries such as scoliosis $(12,13)$. Some centers reported that the use of systemic narcotics cause respiratory depression, epidural local anesthetics strengthen analgesic effect of narcotics (9).

The aim of this study was to investigate and compare the analgesic effects and side effects of epidural infusion of combined local anesthetics and narcotics with epidural infusion of narcotics alone and IV morphine as control group in patients undergoing spondylolisthesis and spinal fixation surgery in order to select appropriate method of pain management after spinal surgery.

\section{Methods and Materials/Patients}

This study was a single-blind randomized controlled trial (the patient was not aware of the type of intervention. The researcher was also unaware of the contents of the pump, but became aware of the pump's contents by controlling the attached label whenever complications occurred), in which analgesic effects and side effects of epidural infusion of fentanyl alone and combined epidural infusion of bupivacaine-fentanyl and IV morphine (control group) were evaluated in postoperative spondylolisthesis. This study received approval of the Ethics Committee of the university and it was registered by Iranian Registry Clinical Trial (IRCT) under the number of IRCT2014031016917N1. It was carried out within nine months from March 2014 in patients with listhesis candidate for spinal fixation in educational remedial center of Poursina in Rasht. 90 patients (three groups of 30 patients) at the age of 18 to 75 years who had no mental disorders, history of hepatic or renal failure and drug addiction were enrolled in a pilot study with ASA class I-III, (ASA class I: healthy patient without organic, biochemical, or psychiatric disease, ASA class II: a patient with mild systemic disease, ASA class III: significant or severe systemic disease that limits normal activity). All patients suffered from lumbar Spondylolisthesis and were candidate for posterior spine fusion surgery. Exclusion criteria were relocation or accidental removal of the epidural catheter, dural damage or tear at the time of surgery and patient's refusal or lack of cooperation at every stage of the study. All patients were examined one day before surgery and were evaluated with the consideration of the presence of neurological deficits. They were explained study implementation method, the way of measuring pain with VAS, medication infusion pump, medication and its possible side effects in epidural way like sensory or motor block and clinical signs of drug poisoning. Before surgery, patients were divided into three groups using block randomization as block size of six (epidural fentanyl intervention group, epidural fentanyl-bupivacaine intervention group and intravenous morphine control group).

In the operating room, two IV lines with IVC No. 18 were applied and electrocardiogram (ECG), non-invasive blood pressure (NIBP) and pulse oximetry were monitored routinely. Normal saline was infused at five $\mathrm{ml} / \mathrm{kg}$ before anesthesia. All patients received one $\mathrm{mg}$ of lorazepam the night before surgery and $0.05 \mathrm{mg}$ of midazolam as premedication on the day of surgery. All patients were exposed to general anesthesia by an anesthetist in the same way in the supine position. The induction of anesthesia was conducted with $0.3 \mu \mathrm{g} / \mathrm{kg}$ of sufentanil, two $\mathrm{mg} / \mathrm{kg}$ of propofol, and $0.5 \mathrm{mg} / \mathrm{kg}$ of atracurium, and the maintenance of anesthesia was achieved with $50 \mu \mathrm{g} / \mathrm{kg} / \mathrm{min}$ of propofol, $0.1 \mu \mathrm{g} / \mathrm{kg} / \mathrm{min}$ of remifentanil and $10 \mathrm{mg}$ of atracurium (every 30 minutes). Then, the mechanical ventilation of lungs with $\mathrm{O} 2$ and $\mathrm{N} 2 \mathrm{O}$ (Fio2, 0.5) was performed following putting the patient in the prone position. In all patients, instrumentation and posterior fusion was performed in T10-L5 level by a single experienced surgeon. The surgery was done with minimal and usual tissue damage that is possible. In the intervention groups, the epidural catheter (Portex_DuraFlexTM epidural catheters $18 \mathrm{G}$; NH) was placed and fixed in midline, a segment upper than the fusion place, and 3-6 cm into the epidural space. The patients were extubated after surgery and were taken to the recovery room. After recovery, patients' consciousness was evaluated with respect to new neurologic deficits and pain. All patients were infused $1 \mathrm{~g}$ of apotel in $100 \mathrm{ml}$ of normal saline at $20 \mathrm{~min}$ before the patient becomes conscious in order to primary pain control aimed at using multi-modal analgesia, and then it was repeated every six hours. For the intervention group of epidural fentanyl (F), the surgeon using CBI+PCA-MR100 CYS pump made by Changzhou medical bioengineering Co PRC infused $100 \mu \mathrm{g}$ of fentanyl and $98 \mathrm{cc}$ of normal saline at the rate of $3-5 \mathrm{ml} / \mathrm{h}$ (equivalent to 3-5 $\mu \mathrm{g}$ of fentanyl per hour, proportional to the patient's weight). In the intervention group of epidural fentanylbupivacaine (FB), $100 \mu \mathrm{g}$ of fentanyl and bupivacaine 
$0.125 \%$ were infused at the rate of $3-5 \mathrm{ml} / \mathrm{h}$ (proportional to the patient's weight). The epidural drug infusion was started after the patient entered the recovery room. Then, the blood pressure, heart rate, respiratory rate, pain severity were registered at the moment of patient's transfer from recovery room to the ward by the anesthesia resident. Again, the above items were measured by the anesthesia resident every six hours for 24 hours. VAS was used to evaluate the severity of pain. If VAS score was more than four, one $\mathrm{mg}$ of IV morphine bolus was administered to patient for postoperative pain control in the control group or intravenous morphine $(\mathrm{M})$. The number of times and the first required dose of morphine bolus were registered. If there were side effects incidences including nausea, vomiting, pruritus, hypotension (systolic blood pressure less than $90 \mathrm{~mm} \mathrm{Hg}$ or blood pressure drop over $20 \%$ of the base pressure, respiratory depression, decreased level of consciousness, motor block, they were informed by the nurse to be decided accordingly. Four mg of IV ondansetron was used if nausea or vomiting occurred. If annoying pruritus and motor block existed, infusion was stopped. The time of infusions reduced if the respiratory rate dropped to less than 10 times. When this rate reached to less than eight, monitoring of arterial oxygen saturation was carried out by pulse oximetry and infusion stopped. In addition, if the respiratory rate reduced to less than five, naloxone was used. VAS, respiratory rate (RR), hemodynamic status, including heart rate (HR), blood pressure (BP) and the need for prescription of additional analgesics and the first time required for prescription of additional analgesics and incidence of side effects including motor block, pruritus, nausea and vomiting, loss of consciousness, respiratory depression and hypotension were recorded and the three groups data were compared and analyzed using SPSS statistical software.

In this study, three groups of 30 patients were used. Data analysis was conducted in January 2015 and Fisher's exacts test, Kruskal-Wallis (since the distribution of VAS scores in research samples did not follow a normal distribution, Kruskal wallis test was used), repeated measure ANOVA statistical tests and SPSS 16 statistical software were used.

\section{Results}

In our study, 90 patients candidate for Listhesis and spinal fixation surgery having the inclusion criteria in the study were enrolled in one of the three groups of 30 patients (control, epidural fentanyl and epidural fentanylbupivacaine) randomly after signing an informed consent form, and the severity of pain, VAS score, systolic and diastolic blood pressure, heart rate and respiratory rate were evaluated. There was no statistical significant difference between the three groups in mean age, weight, sex, ASA class and surgery duration $(p>0.05)$. The mean age in the control group was $47 \pm 17.3$, in the fentanyl group
$53.5 \pm 14.1$ and in fentanyl-bupivacaine group $49.7 \pm 9.8$ years, suggesting that the age of the patients in the groups were statistically the same, and had no significant difference among them $(p=0.208)$. Also, there have been no significant difference in the duration of surgery among the three groups $(p=0.917)$. This duration in the control, fentanyl and fentanyl-bupivacaine groups was $187.33 \pm 41.9,192 \pm 46.3$ and $189.6 \pm 43.1$ minutes, respectively. Furthermore, considering the weight of the patients, it was found that the average weights of the control, fentanyl and fentanylbupivacaine groups were $73.3 \pm 10,75.1 \pm 11.9$ and $77.7 \pm 11.6$ $\mathrm{kg}$, respectively, suggesting that there was no significant statistical difference in weight among the three groups based on ANOVA statistical analysis $(\mathrm{P}=0.327)$. In the distribution of the three groups, there was no statistically significant difference in age. Considering comparison of the distribution of ASA class, in control group, $60 \%$ were at ASA class I, 36.7\% at ASA class II and 3.3\% at ASA class III. In the fentanyl group, $60 \%$ were at ASA class I, $40 \%$ at ASA class II and $0 \%$ at ASA class III. In the fentanylbupivacaine group, $46.7 \%$ were at ASA class I, $53.3 \%$ at ASA class II and $0 \%$ at ASA class III, indicating that there was no statistically significant difference based on the Fisher's exacts test $(\mathrm{P}=0.446)$.

Statistical analysis shows that the mean VAS at the time of patient's transfer to the ward in the control, fentanyl and fentanyl-bupivacaine groups was 5.1 $\pm 2.5,3.9 \pm 2.9$, and $3.8 \pm 2.5$, respectively, indicating that there was no statistically significant difference among the three groups according to the Kruskal-Wallis test. VAS score at 6th hour after the patient's transfer to the ward is as follows: mean VAS based on VAS score was $4.6 \pm 3,5.4 \pm 2.5$ and $3.1 \pm 1.7$ in the control, fentanyl and fentanyl-bupivacaine groups, respectively. This difference among the three groups was statistically significant, so that mean VAS in this period was the least in the fentanyl-bupivacaine group. Additionally, the mean VAS on the basis of VAS score at 12 th hour after patient's transfer to the ward was $4.3 \pm 2.7$, $4.5 \pm 2.1$ and $2.6 \pm 1.9$ in the control, fentanyl and fentanylbupivacaine groups, respectively and these differences were significant according to Kruskal-Wallis test $(\mathrm{P}=0.001)$. The pain severity was the least in fentanyl-bupivacaine group and half the severity of pain in the other two groups. Mean of VAS at 18th hour after patient's transfer to the ward was $3.2 \pm 2.4,3 \pm 2.4$ and $1.9 \pm 1.5$ in the control, fentanyl and fentanyl-bupivacaine groups, respectively. While the mean VAS score in the fentanyl-bupivacaine group was less than that in the other two groups, the difference was not statistically significant $(\mathrm{P}=0.171)$. Considering statistical analysis of mean VAS on the basis of VAS score at 24th hour after patient's transfer to the ward indicates that despite the VAS score in the fentanyl-bupivacaine group is less than that in the other two groups, this difference was not statistically significant. $(\mathrm{P}=0.818)$ (Table 1$)$. 
Table 2: The mean VAS based on VAS Score in Three Groups

\begin{tabular}{|c|c|c|c|c|c|}
\hline \multicolumn{3}{|c|}{ Variables } & Mean & Std. Deviation & $\mathbf{P}$ \\
\hline \multirow{15}{*}{ VAS } & \multirow{3}{*}{ Arrival } & CONTROL & 5.1 & 2.5 & \multirow{3}{*}{0.069} \\
\hline & & FENTANYL & 3.9 & 2.9 & \\
\hline & & FENTANYL+BUPIVACAIN & 3.8 & 2.5 & \\
\hline & \multirow{3}{*}{ After 6 hours } & CONTROL & 4.6 & 3.0 & \multirow{3}{*}{.001} \\
\hline & & FENTANYL & 5.4 & 2.5 & \\
\hline & & FENTANYL+BUPIVACAIN & 3.1 & 1.7 & \\
\hline & \multirow{3}{*}{ After 12 hours } & CONTROL & 4.3 & 2.7 & \multirow{3}{*}{.001} \\
\hline & & FENTANYL & 4.5 & 2.1 & \\
\hline & & FENTANYL+BUPIVACAIN & 2.6 & 2.0 & \\
\hline & \multirow{3}{*}{ After 18 hours } & CONTROL & 3.2 & 2.7 & \multirow{3}{*}{.171} \\
\hline & & FENTANYL & 3.0 & 2.4 & \\
\hline & & FENTANYL+BUPIVACAIN & 1.9 & 1.5 & \\
\hline & \multirow{3}{*}{ After 24 hours } & CONTROL & 2.2 & 2.6 & \multirow{3}{*}{.818} \\
\hline & & FENTANYL & 1.7 & 1.6 & \\
\hline & & FENTANYL+BUPIVACAIN & 1.7 & 1.9 & \\
\hline
\end{tabular}

The comparative study of pain scoring on the basis of VAS score in the three groups showed decrease of $0.5 \pm 3.4$, increase of $1.5 \pm 3.3$ and decrease of $0.7 \pm 2.7$ scores in the control, fentanyl and fentanyl-bupivacaine groups, respectively, from the time of patient's arrival to 6 hours after surgery, indicating that these differences were statistically significant $(\mathrm{P}=0.027)$. It was found that fentanyl-bupivacaine and fentanyl groups had the highest and lowest in reduction of pain severity, respectively (Table 3). Changes in pain severity on the basis of VAS score, between 6th and 12th hour after patient's transfer to the ward showed that these changes were not statistically significant in the three groups $(\mathrm{P}=0.133)$. Changes in the pain severity on the basis of VAS score, between 12th and 18th hour after patient's transfer to the ward demonstrated the decrease of $1.1 \pm 2,1.5 \pm 1.8$ and $0.7 \pm 1.1$ in the control, Fentanyl and Fentanyl-Bupivacaine groups, respectively. These changes were significant according to Kruskal-Wallis test $(\mathrm{P}=0.007)$. (Since the distribution of VAS scores in the samples did not follow a normal distribution, so Kruskal-Wallis test was used). In addition, changes in pain severity on the basis of VAS score, between 18th to 24th hour after patient's transfer to the ward, pain reduction amount was $1 \pm 3.1,1.3 \pm 2.3$ and $0.2 \pm 1.3$ in the control, fentanyl and fentanyl-bupivacaine groups, respectively. These changes were statistically significant $(\mathrm{P}=0.021)$. Reduction amount in pain severity, however, from the arriving time to the ward to the first 24 hours was $2.9 \pm 2.8,2.2 \pm 3.1$ and $2.1 \pm 3.3$ in the control, fentanyl and fentanyl-bupivacaine groups, respectively. This reduction was not statistically significant $(\mathrm{P}=0.406)$ (Table 2$)$.

Table 2. Comparative Study of Pain Scoring based on VAS Score in Three Groups

\begin{tabular}{|c|c|c|c|c|c|c|c|}
\hline \multicolumn{2}{|c|}{ Time Variables } & & Mean & Std. Deviation & Minimum & Maximum & $\mathrm{P}$ \\
\hline \multirow{20}{*}{ Vas } & \multirow{4}{*}{ arrival_6hr } & CONTROL & .5 & 3.4 & -6.0 & 6.0 & \multirow{4}{*}{0.027} \\
\hline & & FENTANYL & -1.5 & 3.3 & -10.0 & 3.0 & \\
\hline & & FENTANYL+BUPIVACAIN & .7 & 2.7 & -4.0 & 8.0 & \\
\hline & & Total & -.1 & 3.3 & -10.0 & 8.0 & \\
\hline & \multirow{4}{*}{$6 \mathrm{hr} \_12 \mathrm{hr}$} & CONTROL & .3 & 1.9 & -5.0 & 5.0 & \multirow{4}{*}{0.133} \\
\hline & & FENTANYL & .9 & 2.2 & -6.0 & 4.0 & \\
\hline & & FENTANYL+BUPIVACAIN & .5 & 1.5 & -3.0 & 3.0 & \\
\hline & & Total & .6 & 1.9 & -6.0 & 5.0 & \\
\hline & \multirow{4}{*}{ 12hr_18hr } & CONTROL & 1.1 & 2.0 & -3.0 & 7.0 & \multirow{4}{*}{0.007} \\
\hline & & FENTANYL & 1.5 & 1.8 & -3.0 & 4.0 & \\
\hline & & FENTANYL+BUPIVACAIN & .7 & 1.1 & .0 & 4.0 & \\
\hline & & Total & 1.1 & 1.7 & -3.0 & 7.0 & \\
\hline & \multirow{4}{*}{ 18hr_24hr } & CONTROL & 1.0 & 3.1 & -7.0 & 8.0 & \multirow{4}{*}{0.021} \\
\hline & & FENTANYL & 1.3 & 2.3 & .0 & 9.0 & \\
\hline & & FENTANYL+BUPIVACAIN & .2 & 1.3 & -3.0 & 3.0 & \\
\hline & & Total & .8 & 2.3 & -7.0 & 9.0 & \\
\hline & \multirow{4}{*}{ arrival_24hr } & CONTROL & 2.9 & 2.8 & -2.0 & 8.0 & \multirow{4}{*}{0.406} \\
\hline & & FENTANYL & 2.2 & 3.1 & -2.0 & 10.0 & \\
\hline & & FENTANYL+BUPIVACAIN & 2.1 & 3.3 & -6.0 & 10.0 & \\
\hline & & Total & 2.4 & 3.1 & -6.0 & 10.0 & \\
\hline
\end{tabular}


Figure 1 showing general changes of pain severity on the basis of VAS score in 24 hour follow-up according to repeated measure ANOVA analysis, demonstrates that the trend of the three groups' changes was not statistically identical, and this difference was significant $(\mathrm{P}=0.02)$. Generally, the severity of pain on the basis of VAS score follows a steady decline after the patient's arriving at the ward in the Fentanyl-Bupivacaine group, but in the Fentanyl group, pain severity increases from the arriving time to the ward to 6th hour afterwards, then after 6 hours, with a sharp decline in the slope, pain severity becomes almost identical with that in the Fentanyl-Bupivacaine group at 24th hour. However, once the patient reaches the ward, the pain severity in the control group is remarkably higher than that in the two other groups and it goes downward with a lower slope to 12th hour and subsequently, the decreasing trend slope increased. But finally, the pain severity in this group was higher than that in the two other groups after 24 hours (Figure 1).

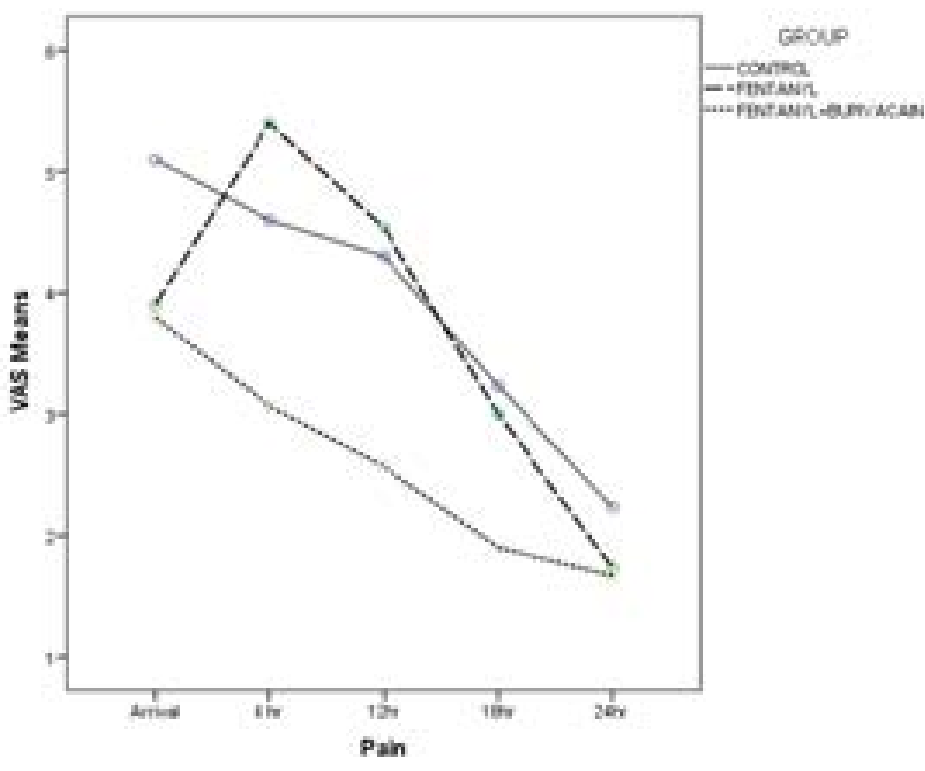

Figure 1: Changes in Severity of Pain based on VAS Score in 24 hour Follow-up in Three Groups

The study of changes in HR (heart rate) based on repeated measure ANOVA analysis showed that HR changes trend during the 24-hour course was not statistically significant. With respect to the values, it was found that HR in the group of fentanylbupivacaine had an increasing trend from the time of arriving at the ward to 12th hour with a fluctuation in HR from 6th to 12th. But In the control group, HR had an almost steady state in the interval between the arriving at the ward to 6th hour, then it decreased at interval between 6th to 12 th hour and then rose again. Conversely, mean HR in fentanyl group had a decreasing trend from arriving at the ward and then, there was a dramatic decrease in HR and it became constant, susequently. However, the changes were not statistically significant $(\mathrm{P}=0.353)$.
Considering RR (respiratory rate), it was shown that there was a steady downward trend in the control group, i.e. the decreasing trend was constant from the time of arriving at the ward to 24 hours afterwards. Although the decreasing trend was seen in the fentanyl group, there was fluctuation between the periods studied in mean RR. The fluctuation in the fentanylbupivacaine group was observed much more than those in the fentanyl group. There was an increasing trend from arriving at the ward to 6th hour and then a decreasing trend between 6 th and 12th hour later. Similarly, the increasing trend was observed between 12th and 18th hour and then the decreasing trend occurred. The difference between this kind of changes with changes in the control and fentanyl groups was statistically significant $(\mathrm{P}=0.0001)$.

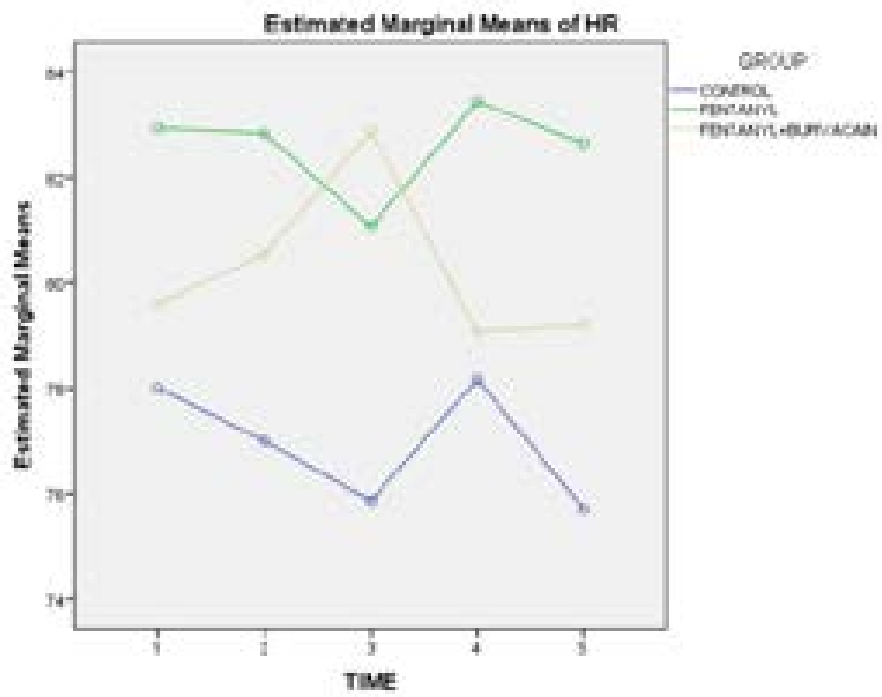

Figure 2: Change in HR (heart rate) in Three Groups in 24 hours

In studying systolic blood pressure, it was shown that the trend of changes in the three groups was not statistically significant $(\mathrm{P}=0.905)$. Although in the fentanyl-bupivacaine group there was a sharper reduction in systolic blood pressure than that in the other two groups at the time of patient's arriving to the ward to 6th hour, the trend of changes was fairly similar.

In the study of changes in diastolic blood pressure within 24 hours, no significant difference was observed $(\mathrm{P}=0.612)$. The type of variations and fluctuation was almost similar in all the three groups.

The percent distribution of nausea and vomiting showed that these symptoms were seen in $16.7 \%$ (five patients) of control group patients, while they were not observed in the two other groups, indicating that the difference was significant on the basis of Fisher's exact test $(\mathrm{P}=0.005)$. However, pruritus, motor block, loss of consciousness and respiratory depression were reported as $0 \%$ in all groups and there was no significant difference $(\mathrm{p}>0.05)$. 


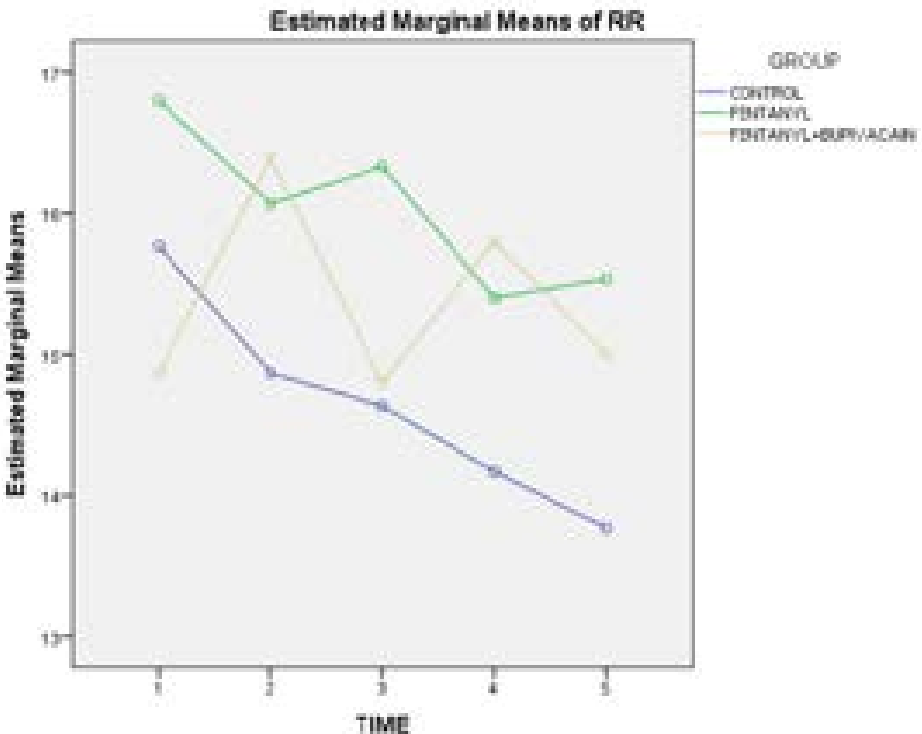

Figure 3: Trend of Changes in RR in Three Groups within 24 hours

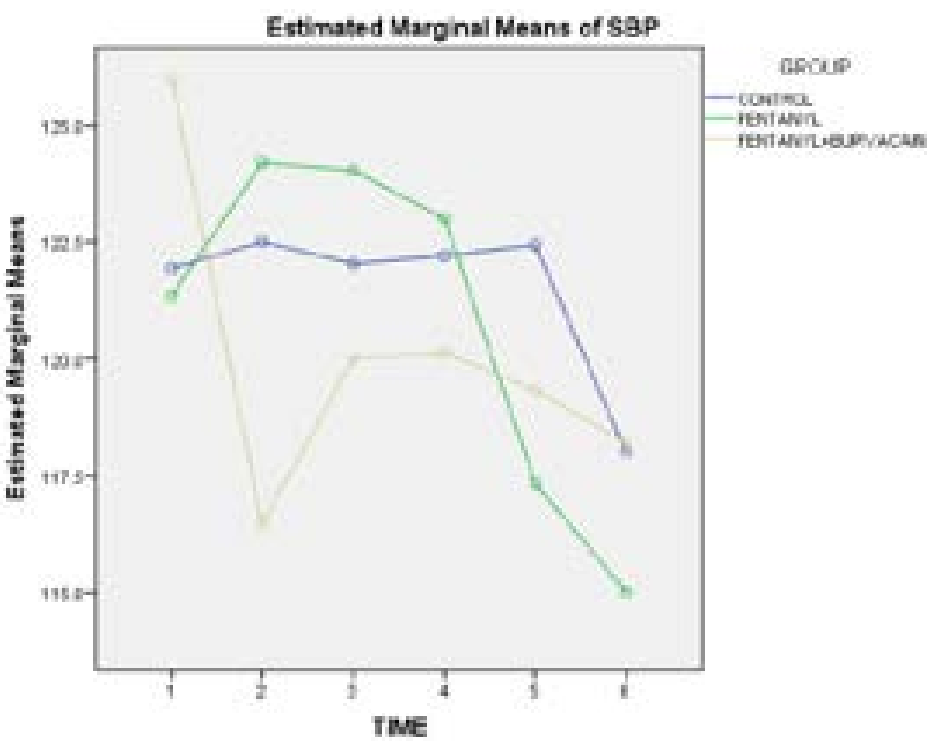

Figure 4: Trend of Changes in Systolic Blood Pressure within 24 hours

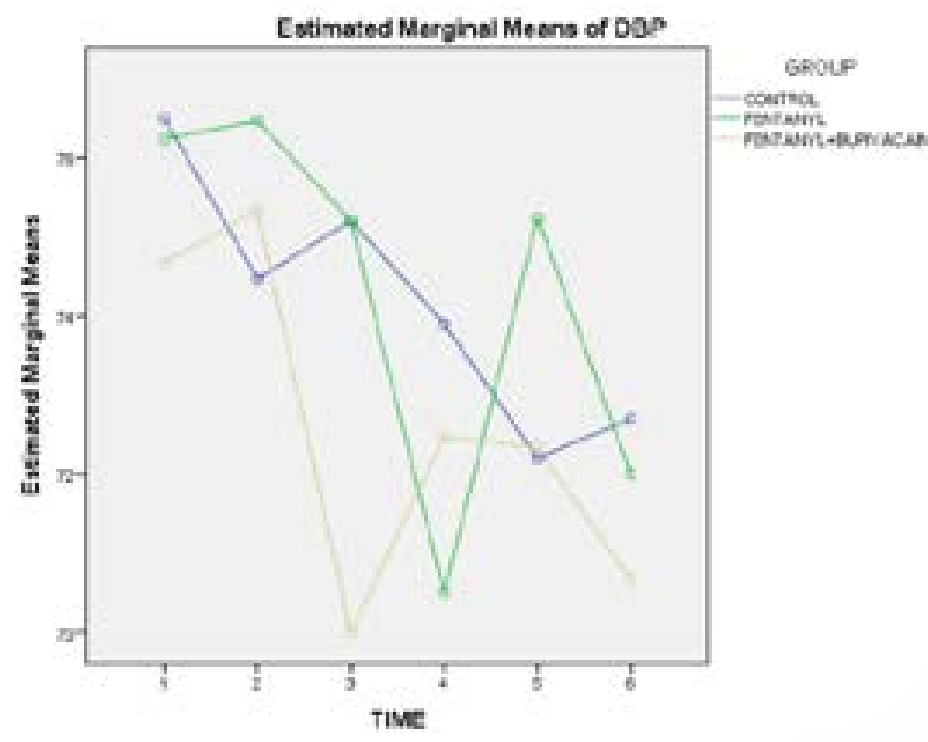

Figure 5: Changes in Diastolic Blood Pressure within 24 hours

\section{Discussion}

We studied and compared three analgesic methods of epidural infusion of fentanyl alone and epidural infusion of fentanyl-bupivacaine and injection of PRN IV morphine on postoperative pain in patients undergoing spondylolisthesis and spinal fixation surgeries. This study showed that patients in the epidural infusion of fentanylbupivacaine experienced less pain significantly, especially in the first 6 hours after surgery. It was very important in the rehabilitation, and the patients were more satisfied than the two other groups. However, evidence of additional side effects such as nausea, vomiting, respiratory depression, motor block, pruritus, loss of consciousness was observed in this group in the study. Since preoperative placement of the epidural catheter may interfere with spinal surgery, intraoperative placement of catheter for postoperative pain control has been proposed (5). Serious complications associated with this procedure are rare. Cassady et al (2000) reported one case of wound infection caused by this procedure that did not have to do with the placement of the catheter during surgery (14). Shaw et al (1996) reported a case of respiratory depression due to receiving high-dose of medication through epidural infusion that led to apnea (15). In our study, no cases of wound infection and respiratory depression were reported. For more safety, patients who had sustained dural damage were excluded from the study because the amount of medication infused into the intrathecal space couldn't be evaluated.

Cohen et al. (1997) compared analgesic effects of epidural infusion of Bupivacaine $0.0625 \%$ with fixed-dose whose catheter was placed two or three levels above the surgery one, during the surgery with IV morphine pump group $(1 \mathrm{mg} / \mathrm{mL})$ in the major surgeries of spine and observed no significant difference in postoperative pain severity between the two groups. In this study, mean VAS in epidural group was $4.5,3.5$ and 3.8 in the first, second, third day after surgery, respectively. Therefore, it may be due to insufficient dose of local anesthetic used in surgical site in this study (16). While in the present study, higher concentration of fentanyl-bupivacaine $(0.125 \%)$ was used, so that it obviously reduced pain in the first 6 hours after surgery compared to epidural fentanyl and IV morphine groups $(\mathrm{P}=0.001)$. In a case series, Lowry et al (2001) showed that after anterior spinal fusion, 10 patients receiving epidural fentanyl, hydromorphone and ropivacaine $0.125 \%$ was $2.3(\mathrm{SD}=0.9)$ on the first postoperative day (13). Yun-Hui Teng et al. (2004) studied retrospectively on 859 patients with a mean age of 64 years undergoing major surgery and compared the analgesic effects of the epidural fentanyl-bupivacaine with IV or epidural form of morphine, and reported that epidural fentanyl-bupivacaine was more effective than intravenous or epidural morphine forms on postoperative acute pain control and had fewer side effects. Nausea and vomiting were observed more frequently in the epidural morphine group $(\mathrm{p}<0.05)$ and pruritus was reported in 
a few patient in Fentanyl-Bupivacaine group $(p<0.05)$. Other complications include urinary retention, sedation and motor block that were similar in the three groups (8). Michael R. Schenk et al. (2006) in a study on 61 patients undergoing spinal fusion surgery compared analgesic effects of PCEA (ropivacaine $0.125 \%$ and $1.0 \mathrm{~g} / \mathrm{mL}$ of sufentanil) at the rate of $14 \mathrm{~mL} / \mathrm{h}$, with the IV form of morphine, and reported that the pain severity in the PCEA group was lower than that in IV group and the patients were more satisfied $(p<0.01)$ (1) Haitham Abou Zeid et al. (2012) in a study on 40 patients undergoing thoracotomy compared the analgesic effects of the intrathecal morphine with paravertebral bupivacaine and came to the conclusion that there was no significant difference between the two groups in the VAS in the first 48 hours postoperatively at rest and during coughing, nausea, pruritus and urinary retention (7). Local anesthetic high concentrations may lead to the motor block that prevents early moving of patients and fast diagnosis of neurological complications associated with surgery. Proper dose and concentration of epidural local anesthetic for pain control after major spinal surgery have not become clear yet. However, no motor block was reported using Bupivacaine $0.125 \%$ in the present study.

\section{Funding}

None declared.

\section{Conflicts of Interest}

The authors declare that they have no competing interests.

\section{References}

1. Michael R. Schenk, Michael Putzier, Bjoern Ku“ gler and et al. Postoperative Analgesia After Major Spine Surgery: Patient-Controlled Epidural Analgesia Versus Patient-Controlled Intravenous Analgesia: Anesth Analg. 2006;103:1311-17.

2. Bianconi $M$, Ferraro $L$, Ricci $R$, et al. The pharmacokinetics and efficacy of ropivacaine continuous wound installation after spine fusion surgery. Anesth Analg. 2004;98:166 -72.

3. Gottschalk A, Freitag M, Tank S, et al. Quality of postoperative pain using an intraoperatively placed epidural catheter after major lumbar surgery. Anesthesiology. 2004;101:175- 80.
4. Blumenthal S, Min K, Nadig M, Borgeat A. Double epidural catheter with ropivacaine versus intravenous morphine: a comparison for postoperative analgesia after scoliosis correction surgery. Anesthesiology. 2005;102:175- 80 .

5. Eilers K, Schenk M, Putzier M, et al. Use of peridural catheters in the treatment of postoperative pain after spinal instrumented fusion-an experience report. Z Orthop Ihre Grenzgeb. 2002;140: 621-5.

6. Sang Hoon Lee, Kyung Hyun Kim, Seong-Mee Cheong,et al; A Comparison of the Effect of Epidura Patient-Controlled Analgesia with Intravenous PatientControlled Analgesia on Pain Control after Posterior Lumbar Instrumented Fusion: J Korean Neurosurg, 2011: 50 : 205-208.

7. Lebovits AH, Zenetos P, O'Neill DK, Cox D, Dubois MY, Jansen LA, et al. Satisfaction with epidural and intravenous patient-controlled analgesia. Pain Med. 2001:2:280-286.

8. Teng YH, Hu JS, Tsai SK, Liew C, Lui PW. Efficacy and adverse effects of patient-controlled epidural or intravenous analgesia after major surgery. Chang Gung Med J. 2004;27:877-886.

9. Kumar RJ, Menon KV, Ranjith TC. Use of epidural analgesia for pain management after major spinal surgery. Journal of Orthopaedic Surgery. 2003:11(1): 67-72.

10. Cata JP, Noguera EM, Parke E, Ebrahim Z, Kurz A, Kalfas I, et al. Patient-controlled epidural analgesia (PCEA) for postoperative pain control after lumba spine surgery. J Neurosurg Anesthesiol 2008;20: 256260.

11. Fisher CG, Belanger L, Gofton EG, Umedaly HS, Noonan VK, Abramson $C$, et al. Prospective randomized clinical trial comparing patient-controlled intravenous analgesia with patient-controlled epidural analgesia after lumbar spinal fusion. Spine. 2003;28:739-743

12. Van Boerum DH, Smith JT, Curtin MJ. A comparison of the effects of patient-controlled analgesia with intravenous opioids versus epidural analgesia on recovery after surgery for idiopathic scoliosis. Spine. 2000;25:2355-2357.

13. Lowry KJ, Tobias J, Kittle D, Burd T, Gaines RW. Postoperative pain control using epidural catheters after anterior spinal fusion for adolescent scoliosis. Spine. 2001;26:1290-3.

14. Cassady JF Jr, Lederhaas G, Cancel DD, et al. A randomized comparison of the effects of continuous thoracic epidural analgesia and intravenous patient-controlled analgesia after posterior spinal fusion in adolescents. Reg Anesth Pain Med. 2000;25:246 -53.

15. Shaw BA, Watson TC, Merzel DI, et al. The safety of continuous epidural infusion for postoperative analgesia in pediatric spine surgery. J Pediatr Orthop. 1996;16:374-7

16. Cohen BE, Hartman MB, Wade JT, et al Postoperative pain control after lumbar spine fusion. Patient-controlled analgesia versus continuous epidural analgesia. Spine. 1997;22:1892-7.

17. Haitham Abou Zeid, Ahsan Khaliq Siddiqui, Ehab F. A. Elmakarem, Yasser Ghonaimy, Awatif A Nafea, Comparison between intrathecal morphine with paravertebral patient controlled analgesia using Bupivacaine for intraoperative and post-thoracotomy pain relief. Saudi journal of anaesthesia 07/2012 6(3):201-6. DOI: 10.4103/1658-354X.101204

\section{Comments}

Ali Ashraf and colleagues designed a randomized controlled trial to find an appropriate method of 24-hour pain management after surgery for lumbar spondylolisthesis (1). They had approval of the Ethics Committee of the Guilan University of Medical Sciences and registration number from Iranian Registry Clinical Trial (IRCT). They had three groups each consists of 30 patients. Group 1 was treated by fentanyl; group 2 by fentanyl-bupivacaine infusion; and group 3 by intravenous morphine injection. Participants were blinded to the post operative analgesic treatment. However, care providers, outcome assessors, and data analyzer were not blinded and these are important biases of the study. Researchers used block randomization technique which has no bias. However, it is not clear if they performed allocation concealment or not. This is another study bias. All three groups had similar age, sex, weight, ASA class (preoperative health situation) and surgery duration. Authors did not mention if they performed any other cointervention or not. Study continued for only 24 hours. Therefore, there was no attrition bias. Primary outcome measure was pain assessed by visual analogue scale (VAS). There was no significant difference at arrival to the recovery room. However, there is a concern about similarity of initial pain scores. Next evaluations showed lesser pain severity in the second group of fentanyl-bupivacaine after 6 and 12 hours, but not after 24 hours. Secondary outcome measures were complications which was similar in all three groups. Finally, authors showed to decrease first 24 hours post operative pain of patients operated for lumbar spondylolisthesis, epidural infusion of fentanyl-bupivacaine is a safe and effective procedure. However, high or unclear risk of bias was seen for allocation concealment, care provider blindness, outcome assessor blindness, co-intervention and questionable similarity of pain in the initial assessment (2).

Vafa Rahimi-Movaghar, MD, Professor of Neurosurgery, Sina Trauma and Surgery Research Center, Tehran University of Medical Sciences, Tehran, Iran

Reference:

1. Ashraf A, Khanjanian G, Yousefzadeh-Chabok Sh, Alijan B, Ebrahimpour N, Chavoshi T. Comparison of Analgesic Effects of Epidural Infusion of Fentanyl and Fentanylbupivacaine in Spine Surgery: A Randomized Clinical Tria Study. IrJNS. 2015;1(1):28-34.

2. Furlan $A D$, Pennick $V$, Bombardier $C$, van Tulder $M$ Editorial Board, Cochrane Back Review Group. 2009 updated method guidelines for systematic reviews in the Cochrane Back Review Group. Spine (Phila Pa 1976). 2009;34(18):1929-41. 